\title{
Transient behavior between multi-cell flow states in ferrofluidic Taylor-Couette flow
}

\author{
Sebastian Altmeyer ${ }^{1}$, Younghae Do ${ }^{2}$, Soorok Ryu ${ }^{2}$ \\ ${ }^{1}$ Castelldefels School of Telecom and Aerospace Engineering (EETAC), Universitat Polytecnica de \\ Catalunya (UPC), Spain \\ 2 Department of Mathematics, KNU-Center for Nonlinear Dynamics, Kyungpook National University, Daegu \\ 41566, South Korea
}

We investigate transient behaviors induced by magnetic fields on the dynamics of the flow of a ferrofluid [1] in the gap between two concentric, independently rotating cylinders. Without applying any magnetic fields, we uncover emergence of flow states constituted by a combination of a localized spiral state in the top and bottom of the annulus and different multi-cell flow states with toroidally closed vortices in the interior of the bulk. However, when a magnetic field is presented, we observe the transient behaviors between multi-cell states passing through two critical thresholds in a strength of an axial (transverse) magnetic field [2]. Numerical simulations are carried out by solving the ferrohydrodynamical equation of motion using the Niklas approximation [3]. Before the first critical threshold of a magnetic field strength, multi-stable states with different number of cells can be observed. After the first critical threshold, we find the transient behavior between the three- and two-cell flow states. For stronger magnetic fields or after the second critical threshold, we discover that multi-cell states to disappear and a localized spiral state remains stimulated in the system.

Without applying any magnetic fields, we found the emergence of two flow states constituted by a combination of a localized spiral state $\left(\mathrm{SPI}_{l}\right)$ in the top and bottom of the annulus and different multi-cell flow states $\left(\mathrm{SPI}_{l+2 v}, \mathrm{SPI}_{l+3 v}\right)$ with toroidally closed vortices in the interior of the bulk $\left(\mathrm{SPI}_{l+2 v}=\mathrm{SPI}_{l}+\mathrm{SPI}_{2 v}\right.$ and $\left.\mathrm{SPI}_{l+3 v}=\mathrm{SPI}_{l}+\mathrm{SPI}_{3 v}\right)$. The appearing of these multi-stable states is based on the initial conditions.

Applying any magnetic field and changing it's strength can trigger transitions among various flow states, for example, the two-cell and three-cell flow states. The emergence of the flow states, dynamical evolution, and transitions among the various flow states can be summarized in detail, as follows. By increasing the axial [transverse] magnetic field strength, we first identify a transition from $\mathrm{SPI}_{l+3 v}\left[\left(\mathrm{SPI}_{l+2 v}\right]\right.$ to $\mathrm{SPI}_{l+2 v}\left[\mathrm{SPI}_{l+3 v}\right]$, respectively. However, for strong enough magnetic fields, we discover the second transition only leaving a $\mathrm{SPI}_{l}$ state behind.

Although the flow states under fairly large magnetic fields $\left(s_{x}\right.$ or $\left.s_{z}\right)$ are $\mathrm{SPI}_{l}$, there is a significant difference between two final $\mathrm{SPI}_{l}$ states. For applying the strong transverse magnetic field $\left(s_{x}\right), \mathrm{SPI}_{l}$ is orientated close to top and bottom lid located in the Ekman vortex regime. But $\mathrm{SPI}_{l}$ under the strong axial magnetic field $\left(s_{z}\right)$ is orientated more towards the center of the bulk. According to the different type of magnetic fields, $\mathrm{SPI}_{l}$ state can move to or away from the Ekman region.

As to expect, the transitions between the multi-cell flow states are always accompanied by a change in the wavelength and wavenumber, respectively. However, in the present study the symmetry breaking effect (a stimulated two-cell mode $[4,5]$ ) of the transverse magnetic field is obviously present, but plays a significant minor role than in other studies. It becomes more and more pronounced for the larger magnetic field strength $s_{x}$ and $s_{z}$. 

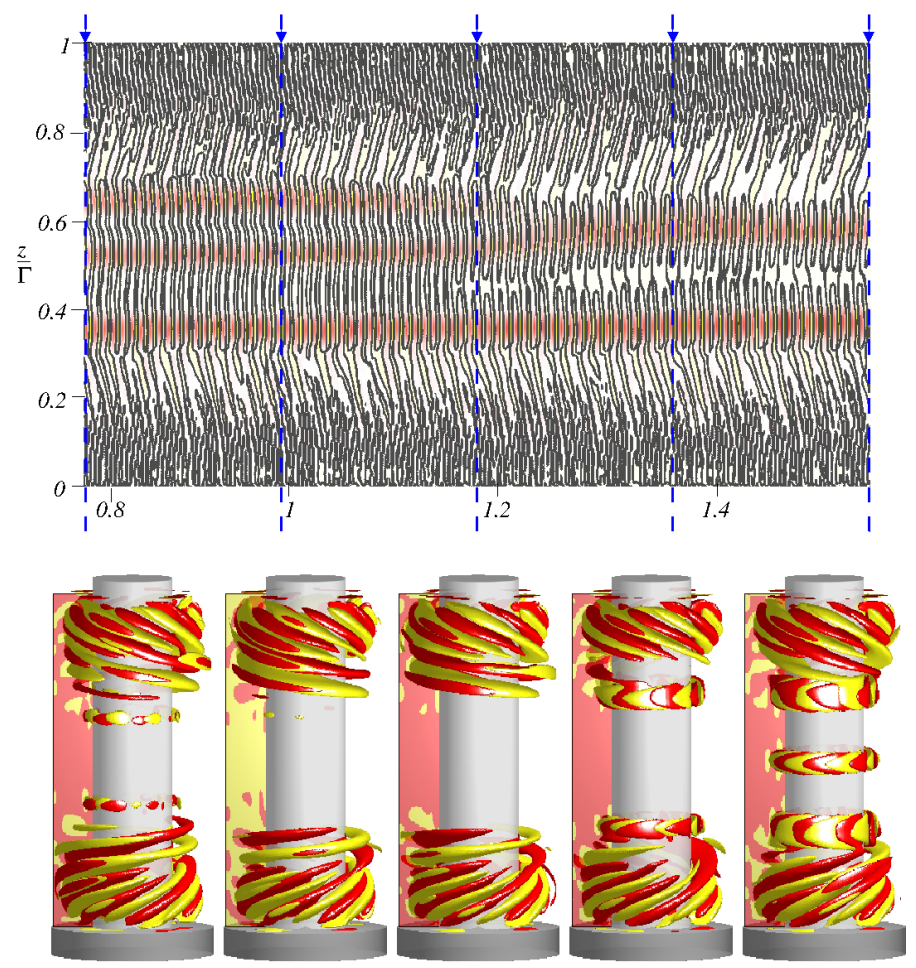

Figure 1: Transitions from $\mathrm{SPIl}_{l+3 V}$ to $\mathrm{SPI}_{l+2 V}$. Top: Space-time plot of the azimuthal vorticity $\eta$ during the transition at $r=r_{1}+0.1 d$. Red (dark gray) and yellow (light gray) correspond to positive and negative values, with $\eta \in[-440,440]$. Bottom: Snapshots of corresponding vortex structures during the transition.

\section{References}

[1] R. E. Rosensweig, Ferrohydrodynamics. Camb. University Press, Cambridge (1985).

[2] S. Altmeyer, Y. Do, and S. Ryu Transient behavior between multi-cell flow states in ferrofluidic Taylor-Couette flow. Chaos 27, 113112 (2018).

[3] M. Niklas, Influence of magnetic fields on Taylor vortex formation in magnetic fluids. Z. Phys. B 68, 493 (1987).

[4] S. Altmeyer, C. Hoffmann, A. Leschhorn, and M. Lücke, Influence of homogeneous magnetic fields on the flow of a ferrofluid in the Taylor-Couette system. Phys. Rev. E 82, 016321 (2010).

[5] M. Reindl and S. Odenbach, Effect of axial and transverse magnetic fields on the flow behavior of ferrofluids featuring different levels of interparticle interaction. Phys. Fluids 23, 093102 (2011). 